\title{
Los vínculos entre estilos de aprendizaje y estrategias de motivación de estudiantes de Grado en Brasil y España
}

\author{
Canós-Darós, L. ${ }^{\text {, }}$ Barbosa da Silva, A. , Perelló Marín, M.R. c , Lima de Araujo \\ Coelho, A.L. ${ }^{\text {, }}$, Santandreu-Mascarell, C. ${ }^{\mathrm{e}}$ \\ ${ }^{a}$ Universitat Politècnica de València, Valencia, Spain, loucada@omp.upv.es, ${ }^{\mathrm{b}}$ Universidade Federal \\ da Paraiba, Joao Pessoa, Brasil, anielson@uol.com.br, ${ }^{\mathrm{c}}$ Universitat Politècnica de València, \\ Valencia, Spain, rperell@upvnet.upv.es, ${ }^{\mathrm{d}}$ Universidade Federal da Paraiba, Joao Pessoa, Brasil, \\ alalcoelho@gmail.com, ${ }^{\mathrm{e}}$ Universitat Politècnica de València, Valencia, Spain, crisanma@omp.upv.es.
}

\section{Reseumen}

En este trabajo se realiza un análisis de los vínculos entre los estilos de aprendizaje y las estrategias de motivación de estudiantes de Grado de Administración y Dirección de Empresas de la Universidade Federal da Paraíba en Brasil y la Universitat Politècnica de València en España. Para ello, se encuestaron a un total de 106 estudiantes de los últimos cursos de Grado utilizando el inventario de los estilos de aprendizaje de David Kolb (1984) y las estratégias de motivación del MSLQ (Motivated Strategies for Learning Questionaire) de Pintrich et al. (1993), que contiene las siguientes componentes y subescalas: Valor (orientación intrinseca, orientación extrínseca, valor de la tarea), Expectativas (control sobre las creencias y autoeficacia) y Emoción (test de ansiedad). Los datos se analizaron obteniendo resultados descriptivos y de comparación de medias con muestras independientes y análisis de varianza.

La primera sección de los resultados presenta el perfil de la muestra de los dos entornos investigados. Además, se muestran los resultados de los estilos de aprendizaje de los estudiantes por país y el desarrollo de las habilidades que forman los estilos. A continuación, se presenta un análisis de las estrategias de motivación. Los resultados indican diferencias en las estratégias de motivación intrínseca y valor de la tarea, así como que los estudiantes brasileños presentan mayores medias para estas dos dimensiones. Los niveles de ansiedad de los estudiantes en los dos países fueron bajos, con un índice de 3,18 para los estudiantes brasileños y 3,11 para los estudiantes españoles, siendo el valor máximo posible 7. Los resultados de la encuesta revelaron que no hay diferencias significativas entre las estrategias de motivación y los estilos de aprendizaje. 
Los vínculos entre estilos de aprendizaje y estrategias de motivación de estudiantes de Grado en Brasil y España

Palabras clave: Estilos de aprendizaje, Estrategias de Motivación, Grado en Administración y Dirección de Empresas, Brasil, España.

\section{Introducción}

Podemos encontrar en la literatura distintos enfoques de investigación sobre el aprendizaje (experimental, psicométrico, fenomenográfico) y diferentes conceptos y definiciones (estilo, estrategia, proceso, hábito, enfoque, predisposición, táctica). Los estilos de aprendizaje se definen como un conjunto de características intelectuales y de personalidad que configuran el modo en que los estudiantes perciben, interactúan y responden frente a situaciones de aprendizaje (Freiberg, Ledesma \& Liporace, 2017). En cualquier caso, una persona sigue un estilo de aprendizaje cuando se enfrenta al reto de aprender, por lo que se considera que el aprendizaje es un proceso activo en el que el protagonista es el propio discente (Gómez \& Gil, 2018).

En este trabajo seguimos la aportación de Kolb (1984:38), que definió el aprendizaje como el proceso de creación del conocimiento a través de la transformación de la experiencia. El conocimiento es, así, consecuencia de la combinación del entendimiento y de la transformación de la experiencia.

Para ello, en el presente trabajo, nos apoyamos en la herramienta desarrollada por Pintrich et al. (1993) acerca de las estratégias de motivación para el aprendizaje, el cuestionario MSLQ (Motivated Strategies for Learning Questionaire). Este cuestionario contiene 15 subescalas diseñadas a partir de las teorías de aprendizaje socio-cognitivas y ampliamente utilizadas como predictores de los resultados académicos (Credé \& Phillips, 2011). Esta herramienta fue diseñada a fin de identificar 3 tipos teóricos de estrategias de aprendizaje (Duncan \& McKeachie, 2005). En primer lugar, las estrategias cognitivas son evaluadas con cuatro subescalas que miden los ensayos del estudiante (repeticiones de la información para sí mismo), elaboración (resumen de la información), organización (de la nueva información a modo de trablas o esquemas), y pensamiento crítico (evaluación de nuevas ideas y su aplicación a situaciones novedosas). Las estrategias metacognitivas son evaluadas por una única subescala que trata de captar la habilidad del estudiante para supervisar sus propios procesos mentales y ajustarlos a sus necesidades, lo cual es un importante componente en el marco auto-regulador del aprendizaje (Pintrich, 2000). Por ultimo, la gestión de recursos se evalúa con cuatro subescalas que tratan de identificar la habilidad del estudiante para gestionar los recursos que tiene disponibles. Estos incluyen el tiempo y el entorno de estudio (habilidades de estudio y uso apropiado del tiempo de estudio), regulación del esfuerzo (persistir con el estudio aunque el material sea aburrido), 
aprendizaje por pares (trabajar con otros y el uso de grupos de estudio), y solicitud de ayuda (pedir ayuda a otros compañeros o profesores) (Credé \& Phillips, 2011).

En este trabajo analizamos las relaciones entre los estilos de aprendizaje y la motivación de aprendizaje de los estudiantes de cursos de administración y gestión de empresas ubicados en dos países: Brasil y España. Además, identificamos si hay diferencias entre los entornos investigados. Nuestra intención es ampliar el conocimiento sobre estos vínculos entre los estilos de aprendizaje y la motivación para ayudar a los profesores a planificar su acción docente basada en una comprensión más amplia.

\section{Metodología}

La investigación tuvo como objetivo analizar los vinculos entre los estilos de aprendizaje y la motivación de aprendizaje de estudiantes de cursos de grado de una universidad pública de Brasil y una de España. El estudio es de naturaleza cuantitativa y descriptiva. A continuación, presentamos el entorno de la investigación, la muestra, los instrumentos de recogida de datos y el proceso de recogida de datos y de análisis.

\subsection{Muestra de la investigación}

La encuesta fue realizada en los grados de Administración y Dirección de Empresas (ADE) de la Universidade Federal da Paraíba (UFPB), en Brasil y de la Universitat Politècnica de València (UPV), en España.

En ella participaron 106 estudiantes de los dos países, 54 de Brasil y 52 de España, matriculados en seis asignaturas de último curso del grado de ADE.

En Brasil, la encuesta fue realizada en las asignaturas Administración de Marketing I (MKT I), Administración de Marketing II (MKT II), Elaboración y Gestión de Proyectos Públicos y Privados (EGPPP) y Gestión Tecnológica del Sector Público y Privado (GTSPP).

En MKT I se trabaja conceptos, evolución y ambientes de marketing; el papel del marketing en las organizaciones y en la sociedad; comportamiento del consumidor y proceso de compra; análisis de mercados consumidores y organizacionales, así como el compuesto de marketing. La asignatura MKT II, se concentra en los temas relacionados con la investigación de marketing, sistemas de información de marketing e inteligencia de marketing, estrategia de marketing, y marketing social.

En EGPPP se trabaja la importancia del proyecto en el proceso de planificación; tipos de proyecto; los orígenes de proyectos (públicos y privados), las etapas de elaboración de un proyecto; estudios de mercado y técnicas de medición de oferta y demanda; definición de la 
Los vínculos entre estilos de aprendizaje y estrategias de motivación de estudiantes de Grado en Brasil y España

demanda y de la ingeniería del proyecto; aspectos locales; análisis de viabilidad económica y financiera; medidas de control y monitoreo; el cierre y los instrumentos de evaluación de proyectos. En GTSPP, se trabaja el papel de la información y la tecnología de la información (TI) en las organizaciones modernas en general y en las agencias gubernamentales en particular desde una perspectiva organizativa de la tecnología organización de los servicios públicos y privados y los procesos de toma de decisiones, y negocios electrónicos (e-business).

En el caso de los estudiantes de España todos estaban matriculados en alguna las siguientes asignaturas: Marketing en Empresas de Servicios Profesionales (MESP) y Sistemas de Control Estratégico (SCE).

En MESP, los alumnos profundizan en las peculiares de las empresas de servicios profesionales. De este modo, partiendo de los conocimientos previos adquiridos en asignaturas de cursos anteriores, trabajan las 8 Ps del marketing mix de servicios, y desarrollan en detalle un plan de marketing de una empresa real.

Por otro lado, en SCE los alumnos aprenden conceptos relacionados con el control estratégico de las organizaciones, es decir, el proceso mediante el cual la dirección influye sobre el resto de la empresa para desarrollar las estrategias y objetivos de la misma. Se trabajan principalmente tres dimensiones: la estrategia, la operativa y la económica, considerando como núcleo el sistema de información de la empresa.

\subsection{Instrumentos de la encuesta}

Para alcanzar el objetivo de la investigación, los instrumentos para la recogida de datos fueron el inventario de los estilos de aprendizaje de David Kolb (1984) y la motivación de aprendizaje del MSLQ (Motivated Strategies for Learning Questionaire) de Pintrich et al. (1993).

El inventario de Kolb (KLSI - Kolb's Learning Style Inventory) contiene 12 sentencias, con cuatro alternativas y para cada una de las sentencias el que responde debe indicar su respuesta con el valor 1 para el complemento de la sentencia que representa la manera menos probable de aprender hasta 4 para el complemento de la sentencia que describe mejor cómo aprende. Los valores para cada sentencia no pueden ser repetidos. Las palabras correspondientes a cada una de las sentencias están vinculadas a una de las habilidades de aprendizaje. Después de rellenar las doce sentencias, la puntuación para cada una de ellas es asociada a cada una de las habilidades de acuerdo con las orientaciones propuestas para análisis del inventario.

La determinación del estilo de aprendizaje predominante es definida por medio de la sustracción de las puntuaciones totales de las habilidades de Experiencia Concreta y Conceptualición Abstracta (CA - EC) y de las habilidades de Observación Reflexiva y 
Experimentación Activa (EA - OR). El estilo de aprendizaje predominante es el resultado de los dos valores de sustracción (Kolb \& Kolb, 2008).

El MSLQ es un cuestionario de administración colectiva que evalúa la motivación para el aprendizaje y las estrategias de aprendizaje. En esta investigación solo utilizamos la escala de motivación con 25 ítems, 11 de ellos sobre Valor (orientación intrínseca, orientación extrínseca y valor de la tarea), 10 sobre Expectativa (control sobre creencias y autoeficacia) y 4 sobre la Emoción (Test de ansiedad). Para atribuir las respuestas a cada uno de los ítems, la escala tipo Likert contiene siete puntos en que los estudiantes deben indicar su nivel de acuerdo o desacuerdo para cada una de las afirmaciones. Así, los valores más próximos a uno indican desacuerdo y los valores más próximos a siete expresan acuerdo con relación a la descripción de la afirmación.

\subsection{Recogida de los datos y análisis}

El proceso de recogida de datos en los dos países ocurrió en los meses de febrero y marzo de 2018. Los investigadores explicaron a los estudiantes los objetivos de la encuesta y solicitaron que rellenasen el cuestionario siguiendo las instrucciones presentadas en el formulario de la encuesta.

Después de la recogida de los datos en los dos países, las respuestas de los estudiantes fueran introducidas en un archivo Excel por los investigadores. Después, las bases de datos fueron integradas en un archivo en el software SPSS, versión 22.0. En una primera etapa del análisis, fue realizado un análisis exploratorio de los datos para caracterizar el perfil de la muestra de cada país, los estilos de aprendizaje predominantes, así como los niveles de desarrollo de las habilidades que contribuyen para la delimitación del estilo predominante. A continuación, el nivel de desarrollo de motivación de aprendizaje fue identificado.

La segunda etapa del análisis tuvo como objetivo analizar las relaciones entre las variables investigadas. Para analizar las diferencias entre los países, fue utilizado el test t para muestras independientes. Las variables dependientes analizadas fueron el género, edad, experiencia de trabajo, media de horas dedicadas al estudio por semana, habilidades que determinan los estilos de aprendizaje y la motivación de aprendizaje. Para analizar la homogeneidad de las varianzas evaluamos los resultados del test de Levene. Para las habilidades, motivación de aprendizaje y las horas dedicadas al estudio por semana, la significancia asociada al test fue superior a 0,05. En estos casos, utilizamos los valores del test t para varianzas iguales asumidas entre los dos países. En relación con las variables género, edad y experiencia de trabajo, las varianzas fueran inferiores a 0,05. Así, para estas variables, asumimos que los datos no fueron homogéneos. 
Los vínculos entre estilos de aprendizaje y estrategias de motivación de estudiantes de Grado en Brasil y España

Para evaluar las relaciones entre los cuatro estilos de aprendizaje y la motivación de aprendizaje fue realizado un análisis de varianza (ANOVA) para verificar si hay diferencia entre las dos variables evaluadas a partir de las siguientes hipótesis:

H0: No hay diferencia significativa entre motivación de aprendizaje de los estudiantes con relación a los estilos predominantes de aprendizaje.

H1: Hay diferencia significativa entre la motivación de aprendizaje de los estudiantes con relación a los estilos predominantes de aprendizaje.

\section{Análisis y discusión de resultados}

En nuestra encuesta, el principal interés es conocer las especificidades de los estilos de aprendizaje de los estudiantes brasileños y españoles para percibir si ellos tienen similitudes en sus preferencias en aprender. La tabla 1 presenta una caracterización de la muestra investigada en los dos países.

Tabla 3.1. Estilos de aprendizaje por país

\begin{tabular}{|c|c|c|c|c|}
\hline \multirow{2}{*}{ Estilo de aprendizaje } & \multicolumn{2}{|c|}{ Brasil } & \multicolumn{2}{|c|}{ España } \\
\hline & $\mathbf{N}$ & $\%$ & $\mathbf{N}$ & $\%$ \\
\hline Acomodador & 8 & 14,8 & 3 & 5,8 \\
\hline Divergente & 3 & 5,6 & 2 & 3,8 \\
\hline Asimilador & 27 & 50,0 & 15 & 28,9 \\
\hline Convergente & 16 & 29,6 & 32 & 61,5 \\
\hline Total & 54 & 100,0 & 52 & 100,0 \\
\hline
\end{tabular}

Los resultados indican que los estudiantes españoles tienen un estilo predominantemente convergente $(61,5 \%)$, mientras que en Brasil los estudiantes encuestados son asimiladores $(50,0 \%)$ preferencialmente. Eso indica que la mayoría de los estudiantes brasileños son más teóricos y los estudiantes españoles más pragmáticos.

En este trabajo nuestro propósito es medir cómo estudiantes brasileños y españoles perciben los diferentes tipos de habilidades de estudio y estrategias de aprendizaje además de su planificación y esfuerzo para desarrollar las tareas vinculadas a su formación universitaria y también sobre el aprendizaje colaborativo y búsqueda de soporte en su 
proceso de aprendizaje en el título de Administración y Dirección de Empresas de las dos universidades. La tabla 3.2 presenta los resultados con las puntuaciones medias de los estudiantes de cada país y los resultados de las diferencias de medias (test t) para muestras independientes.

Tabla 3.2. Motivación de Aprendizaje

\begin{tabular}{l|c|c|c|c|c|c}
\hline \multirow{2}{*}{ VALOR } & \multicolumn{2}{c|}{ Brasil } & \multicolumn{2}{c|}{ España } & \multicolumn{2}{c}{ Teste $\mathrm{t}$} \\
\cline { 2 - 7 } & $\mathrm{M}$ & $\mathrm{DT}$ & $\mathrm{M}$ & $\mathrm{DT}$ & $\mathrm{t}$ & Sig. \\
\hline Orientación Intrínseca & 5,44 & 0,92 & $\mathbf{4 , 7 9}$ & 1,27 & $-3,046$ & 0,03 \\
\hline Orientación Extrínseca & $\mathbf{4 , 3 7}$ & 1,10 & 4,33 & 1,12 &,- 171 & 0,87 \\
\hline Valor de La Tarea & 5,93 & 1,00 & $\mathbf{4 , 7 0}$ & 1,41 & $-5,193$ & 0,00 \\
\hline \multicolumn{1}{c|}{ EXPECTATIVA } & & & & & & \\
\hline Control sobre creencias & $\mathbf{5 , 3 6}$ & 0,92 & 5,03 & 1,15 & $-1,637$ & 0,11 \\
\hline Autoeficacia & $\mathbf{5 , 3 7}$ & 0,96 & 5,43 & 0,72 & 0,399 & 0,691 \\
\hline \multicolumn{1}{c}{ EMOCIÓN } & & & & & & \\
\hline Test de Ansiedad & $\mathbf{3 , 1 8}$ & 1,12 & 3,11 & 0,95 & $-0,333$ & 0,74 \\
\hline
\end{tabular}

$\mathrm{M}=$ Media / DT: Desviación Típica

Los resultados de las estrategias cognitivas y metacognitivas presentan puntuaciones medias superiores a 4 en los dos países en cuatro estrategias de aprendizaje, y valores inferiores a cuatro para la estrategia de autorregulación metacognitiva. A pesar del test de diferencias de medias no se apuntan diferencias significativas con niveles de significatividad debajo de 0,05. Las puntuaciones medias de las estrategias de elaboración, pensamiento crítico y autorregulación metacognitiva son más elevadas hacia los estudiantes brasileños, pero las estrategias de repaso y organización de los estudiantes españoles presentan medias superiores.

Un punto relevante de los resultados en los dos países es el bajo valor medio de las estrategias de autorregulación metacognitiva, que están vinculadas al control y regulación cognitiva por los estudiantes y que objetiva la medida del uso de tres estrategias metacognitivas: planeamiento (definición de objetivos y análisis de las tareas), control (seguimiento de atención y comprensión) y regulación de las actividades (ajuste continuo de las actividades) (Tock \& Moxley, 2017; Duncan \& McKeachie, 2005). Esta es una estrategia de aprendizaje relevante porque ayuda a los estudiantes a mejorar su desempeño en su proceso de formación. Los resultados de la encuesta muestran que esa estrategia presentó las puntuaciones medias más bajas en una escala de 1 a 7 , indicando que los estudiantes necesitan mejorar la planificación, control y regulación cognitiva de las actividades. 
Los vínculos entre estilos de aprendizaje y estrategias de motivación de estudiantes de Grado en Brasil y España

\section{Conclusiones}

La realización de una investigación en dos países sobre el tema del aprendizaje presenta algunos retos. Primero, las diferencias culturales entre los estudiantes son caracterizadas por la vivencia de experiencias que sufren la influencia del entorno social, económico, histórico y demográfico. Segundo, las políticas educacionales de los países pueden presentar algunas especificidades en los procesos de aprendizaje lo que influencia los procesos cognitivos de aprendizaje. A pesar de las diferencias, comprender los procesos de aprendizaje de los estudiantes de países diferentes también puede revelar algunas similitudes importantes sobre todo en cursos de formación más aplicados como el de Administración y Dirección de Empresas.

Los resultados de la encuesta realizada con estudiantes de Brasil y España indican que hay diferencias entre los estilos predominantes de aprendizaje. Mientras los estudiantes brasileños son predominantemente asimiladores, los españoles son predominantemente convergentes. Los estudiantes brasileños son más reflexivos y les gustan trabajar más en pareja y equipo que a los españoles.

Sobre las estrategias de aprendizaje, los resultados no apuntaron diferencias significativas entre las estrategias cognitivas y metacognitivas, pero la baja media de las estrategias de autorregulación metacognitivas en los dos países es preocupante, una vez que son las menos desarrolladas, lo que indica una baja capacidad de los estudiantes de regular y controlar sus procesos cognitivos (Pintrich et al., 1993) de planificación, búsqueda de atención, comprensión y el ajuste de las actividades.

Además, ¿cómo pueden los profesores contribuir para el desarrollo de las estrategias de autorregulación de los estudiantes? ¿En qué medida el bajo nivel de desarrollo de las estrategias de autorregulación afecta al desempeño académico de los estudiantes? Futuros estudios pueden analizar estas cuestiones.

La única estrategia de aprendizaje que presentó diferencias significativas entre los dos países fue la organización del tiempo y el ambiente de estudio. Los estudiantes españoles manejan mejor el tiempo y el ambiente de estudio y dedican mas horas de estudio por semana fuera de clase que los brasileños. Una observación significativa y que necesita de mejor análisis en futuros estudios es la relación entre el turno de estudio (diurno o nocturno) en la organización del tiempo y el ambiente de estudio.

Esta investigación presenta algunas limitaciones. Primero, el bajo número de estudiantes con estilos acomodadores y divergentes, comparados con los asimiladores y convergentes, puede haber limitado algunas relaciones. Este es un resultado que merece la atención de los investigadores porque la media de la experiencia concreta fue la más baja de todas las 
habilidades, lo que influencia directamente en la predominancia de los estilos divergentes y acomodadores. Así, uno de los retos en los procesos de enseñanza es estimular a los estudiantes a vivir nuevas experiencias con más apertura y sin preconceptos para mejorar sus niveles de desarrollo de la experiencia concreta.

Otra limitación es el número de la muestra de los dos países. Así, ampliar la muestra es una estrategia para mejorar su representatividad con relación a la población de los estudiantes de los cursos de administración de las dos universidades.

No obstante, este estudio presenta contribuciones para la práctica docente porque indica la importancia de analizar las estrategias de aprendizaje, las habilidades y estilos de aprendizaje de los estudiantes para planear estrategias de enseñanza centradas en el estudiante para mejorar sus capacidades cognitivas y ayudar los estudiantes a administrar mejor sus estudios. También puede ser considerado un trabajo innovador porque su entorno de investigación involucra estudiantes de dos países.

\section{Agradecimientos}

Sistemas de aprendizaje en acción en el ámbito de la educación superior (PIME/2017/A07), Universitat Politècnica de València.

\section{References}

Credé, M., \& Phillips, L. A. (2011). A meta-analytic review of the Motivated Strategies for Learning Questionnaire. Learning and individual differences, 21(4), 337-346.

Duncan, T. G., \& McKeachie, W. J. (2005). The making of the motivated strategies for learning questionnaire. Educational psychologist, 40(2), 117-128.

Freiberg Hoffmann, A., Ledesma, R., \& Fernández Lipporace, M. (2017): Estilos y estrategias de aprendizaje en estudiantes universitarios de Buenos Aires. Revista de Psicología, 35(2), 535-573.

Gómez Ruiz, P. \& Gil López, A.J. (2018). El estilo de aprendizaje y su relación con la evaluación entre pares. Revista de Investigación Educativa, 36(1), 221-237.

Kolb, A.Y. \& Kolb, D.A. (2008). The learning way: meta-cognitive aspects of experiential learning, Simulation \& Gaming, 4(3), 297-327.

Kolb, D.A. (1984). Experiential Learning: experience as the source of learning and development. Englewood Cliffs: Prentice-Hall.

Pintrich, P. R. (2000). The role of goal orientation in self-regulated learning. In Handbook of self-regulation, 451-502. 
Los vínculos entre estilos de aprendizaje y estrategias de motivación de estudiantes de Grado en Brasil y España

Pintrich, P. R., Smith, D. A., Garcia, T., \& McKeachie, W. J. (1993). Reliability and predictive validity of the Motivated Strategies for Learning Questionnaire (MSLQ). Educational and psychological measurement, 53(3), 801-813.

Tock, J. L., \& Moxley, J. H. (2017). A comprehensive reanalysis of the metacognitive selfregulation scale from the MSLQ. Metacognition Learning, 12, 79-111. 